\section{Falls in older adult psychiatric inpatients}

Falls are the commonest cause of trauma in older adults and are of particular concern in hospital settings (Ojo et al., 2009). Osteoporotic fractures occurring as a consequence of falls are a leading cause of morbidity and mortality in this population (Stubbs et al., 2009). Older adult psychiatric long-stay patients are at elevated risk of osteoporosis, with one recent study confirming this link by demonstrating that over half had osteoporosis $(58 \%)$ with just under one-third having ostetopenia (32\%) (Stubbs et al., 2009). The incidence of falls is known to be higher in psychiatric settings, and this is particularly so in older adult settings (Blair and Gruman, 2006). Risk factors predisposing older adult psychiatric patients to falls include chronic illness, high rates of cognitive disturbance, psychotropic medication use and behavioral manifestations, and in particular agitation and wandering, two factors which are both strongly associated with falls (Blair and Gruman, 2006). Clearly, older adult psychiatric patients are at an elevated risk of falls (Blair and Gruman, 2006). Even if a patient has not fallen prior to hospitalization, the mere presence of being admitted immediately increases their subsequent falls risk (Blair and Gruman, 2006).

There is a plethora of research investigating and reporting the incidence and consequence of falls in general older adult settings. Research investigating this phenomenon in older adult psychiatric units is relatively uncommon. This is surprising, especially when one considers the elevated risk of both falls and osteoporotic fractures in this population (Blair and Gruman, 2006; Stubbs et al., 2009). One study (Blair and Gruman, 2006) reported that over a sixmonth period, 28 older adult psychiatric inpatients patients fell, with a total of 45 falls being recorded in that period. In order to add to this limited literature, a retrospective audit was conducted of official hospital accident and incident falls in the Townsend Division of St Andrews Healthcare, a leading innovator in mental health in the U.K. Within this service, there are over 90 beds for older adults with a history of challenging and/or offending behavior. All falls are recorded, regardless of whether an injury occurred. This service has a proactive approach to managing both falls and osteoporosis, with the endeavors relating to the latter recently being reported in the literature (see Stubbs et al., 2009).

Falls data were analyzed over a six-month period across the five wards in the Townsend Division, from December 2007 to June 2008. In this period, there were 303 recorded falls (range per month $32-70$, mean $=50.5$ per month), with 51 patients having at least one recorded fall (range 1-9, mean 5.9). The large majority resulted in no injury ( $\mathrm{n}=229,76 \%)$ with just fewer than a quarter causing injury to patients $(74,24 \%)$. The number of injuries of unspecified severity varied across the five wards from 4 to 26 (mean 14.8). Interestingly, no falls resulted in osteoporotic fracture, and all falls resulted in minor injuries. Just under a half of the falls $(149,49 \%)$ occurred during therapy hours, i.e. between 0800 and 1700 .

The data reported in this research letter add to the limited literature on falls in old age psychiatric patients. This is an "at risk" group for both falls and osteoporosis, which are associated with much mortality and morbidity (Stubbs et al., 2009). This research letter reports that over three-quarters of falls resulted in no injury. When one considers the elevated risk of both falls and osteoporosis in this group, this finding is surprising. Future prospective studies investigating falls and multidisciplinary interventions that seek to reduce the risk of falls should be conducted in an attempt to help increase our knowledge of reducing the risk of falls and subsequent osteoporotic fractures.

\section{References}

Blair, E. and Gruman, C. (2006). Falls in an inpatient geriatric psychiatric population. Fournal of the American Psychiatric Nurses Association, 11, 351-354.

Ojo, P., O'Conner, J., Kim, D., Ciardellio, K. and Bonadies, J. (2009). Pattern of injury in geriatric falls. Connecticut Medicine, 73, 139-145.

Stubbs, B., Zapata, E. and Haw, C. (2009). Screening for osteoporosis: a survey of older psychiatric inpatients at a tertiary referral centre. International Psychogeriatrics, 21, $180-186$.

BRENDON STUbBs, Lead Physiotherapist

Townsend Division, St Andrews Healthcare, Northampton, U.K.

Email: bstubbs@standrew.co.uk 